\title{
Putrescine analogue cytotoxicity against Trypanosoma cruzi
}

Received: 30 June 2005 / Accepted: 23 August 2005 / Published online: 11 November 2005

(C) Springer-Verlag 2005

\begin{abstract}
Trypanosoma cruzi is the etiological agent of American trypanosomiasis. Most of the available data on trypanosomatid parasites were obtained from African trypanosomes. Parasitic protozoa polyamine metabolism and transport pathways comprise valuable targets for chemotherapy. T. cruzi cannot synthesize putrescine, but its uptake from the extracellular milieu can promote parasite survival. Nevertheless, little is known about the cell biology of this diamine in T. cruzi. Here we notice that the putrescine analogue 1,4-diamino-2-butanone (DAB) inhibited T. cruzi epimastigotes' in vitro proliferation and produced remarkable mitochondrial destruction and cell architecture disorganization, as assessed by transmission electron microscopy. Mitochondrial damage was confirmed by MTT reduction. We decided to analyze the oxidative stress undergone by DAB-treated parasites. Thiobarbituric-acid-reactive substances were measured to assess lipid peroxidation. Analogue effects were dosedependent; $5 \mathrm{mM}$ DAB only slightly enhanced peroxidation, whereas $10 \mathrm{mM}$ DAB significantly $(P<0.05)$ diminished it. These data indicate that putrescine uptake by this diamine auxotrophic parasite may be important for epimastigote axenic growth and cellular organization.
\end{abstract}

D. Menezes · M. A. Vannier-Santos $(\bowtie)$

Centro de Pesquisas Gonçalo Moniz,

Fundação Oswaldo Cruz-FIOCRUZ,

Rua Waldemar Falcão 121,

40.295-001 Brotas, Salvador, Brazil

e-mail: vannier@cpqgm.fiocruz.br

Tel.: +55-71-31762236

Fax: $+55-71-31762326$

C. Valentim

Instituto de Biofísica Carlos Chagas Filho,

Universidade Federal do Rio de Janeiro,

Rio de Janeiro, Brazil

\section{F. Oliveira}

Instituto de Bioquímica Médica,

Universidade Federal do Rio de Janeiro,

Rio de Janeiro, Brazil
Keywords Trypanosoma cruzi Putrescine · Polyamine · 1,4-Diamino-2-butanone $\cdot$ Oxidative stress ·

Mitochondria $\cdot$ Electron microscopy $\cdot$ Protozoan

\section{Introduction}

Trypanosoma cruzi is the etiological agent of Chagas disease or American trypanosomiasis, which affects 16-18 million people in South America and Central America and is emerging in the Brazilian Amazon (Coura et al. 2002). Patients may present cardiomyopathy, which has high morbidity and mortality. Treatment of Chagas disease is based on the use of nitroheterocyclic agents, such as nifurtimox and benznidazole ( $N$-benzyl-2-nitro-1-imidazole acetamide), which are associated with considerable side effects and refractory cases (Coura 1988; Gorla et al. 1988). In addition, sterile cures are usually not achievable. Therefore, new antitrypanosomal agents are required; however, despite the high incidence and mortality of the infection, there has been little commercial interest in developing new trypanocidal compounds (Trouiller et al. 2002).

Polyamines are fundamental for growth, development, cellular differentiation, and macromolecule stabilization, among other processes (Pegg and McCann 1982). These polycation biosynthesis pathways in parasitic protozoa differ from those in mammalian cells and, therefore, comprise valuable targets for chemotherapy (Müller et al. 2001; Bacchi and Yarlett 2002). The ornithine decarboxylase (ODC) irreversible inhibitor D,L- $\alpha$-difluoromethylornithine (DFMO) was shown to be effective against both experimental Trypanosoma brucei infections (Bacchi et al. 1980) and against sleeping sickness caused by Trypanosoma gambiense (Marton and Pegg 1995). The selectivity of DFMO on T. brucei and T. gambiense is due to the slow turnover ( $\sim 6 \mathrm{~h}$ half-life) of ODC compared to that of the human enzyme ( $\sim 15$ min half-life).

T. cruzi is devoid of ODC activity (Ariyanayagam and Fairlamb 1997) and thus must incorporate polyamines either from the extracellular milieu or from the host cell by 
means of a high-affinity diamine transport system (Le Quesne and Fairlamb 1996). Putrescine uptake from the host cell may promote $T$. cruzi intracellular proliferation (Freire-de-Lima et al. 2000; Peluffo et al. 2004).

Therefore, putrescine transport and metabolism are of great interest for the understanding of the infection and the chemotherapeutic drug design. The putrescine analogue 1,4-diamino-2-butanone (DAB) inhibits the in vitro proliferation of the trichomonad Tritrichomonas foetus (Reis et al. 1999), Entamoeba invadens (Calvo-Méndez et al. 1993), and Entamoeba histolytica (Arteaga-Nieto et al. 1996), as well as of Leishmania amazonensis (Valentim et al., unpublished) and fungi (Martinez-Pacheco et al. 1989; Ueno et al. 2004), comprising a tool to approach putrescine's role in pathogens. Here we report the effects of this compound on the putrescine auxotroph T. cruzi.

\section{Materials and methods}

Chemicals DAB (1,4-diamino-2-butanone dihydrochloride), putrescine (1,4-diamino-butane or 1,4-tetramethylene-diamine), MTT [3-(4,5-dimethyl-2-thiazolyl)-2,5-diphenyltetrazolium bromide], and thiobarbituric acid were purchased from Sigma (St. Louis, MO, USA). All reagents used were of analytical grade.

Parasites Epimastigotes of T. cruzi Y-strain were cultured at $28^{\circ} \mathrm{C}$ in plastic flasks containing $5 \mathrm{ml}$ of liver infusion trypticase medium inoculated with $10^{7}$ cells $/ \mathrm{ml}$ and supplemented with $10 \%$ fetal calf serum, $100 \mu \mathrm{g} / \mathrm{ml}$ penicillin, and $100 \mu \mathrm{g} / \mathrm{ml}$ streptomycin. Cells from the mid-log phase were harvested by centrifugation at $1,000 \times g$ and washed three times with phosphate-buffered saline (PBS) before the experiments. Parasite growth was assessed in hemocytometric chambers by daily counting under phase contrast microscopy.

Transmission electron microscopy Parasites were fixed in $2.5 \%$ glutaraldehyde (EM-grade; Sigma) and $5 \mathrm{mM} \mathrm{CaCl}_{2}$ in $0.1 \mathrm{M}$ sodium cacodylate buffer ( $\mathrm{pH}$ 7.2); postfixed in $1 \%$ osmium tetroxide and $0.08 \%$ potassium ferricyanide; dehydrated in an acetone series; and embedded in Polybed resin (Polysciences, Inc.). Thin sections were stained with uranyl acetate and lead citrate, and then observed under a Zeiss CEM900 transmission electron microscope.

Lipid peroxidation Thiobarbituric acid-reactive substances (TBARS) were measured in parasites preincubated or not with the analogue for $24 \mathrm{~h}$. After treatment, the cells were washed and resuspended in $200 \mu \mathrm{l}$ of PBS, and then $200 \mu \mathrm{l}$ of $1 \%$ thiobarbituric acid in acetic acid was added. After that, the samples presenting equal cell numbers were incubated at $95^{\circ} \mathrm{C}$ for $3-4 \mathrm{~h}$ and then cooled. Absorbance was determined at $532 \mathrm{~nm}$.

MTT reduction Parasites $\left(10^{7}\right.$ cells $\left./ \mathrm{ml}\right)$ were treated as described above, washed, reinoculated in fresh culture medium containing $10 \% \mathrm{vol} / \mathrm{vol} \mathrm{MTT}$, and incubated for

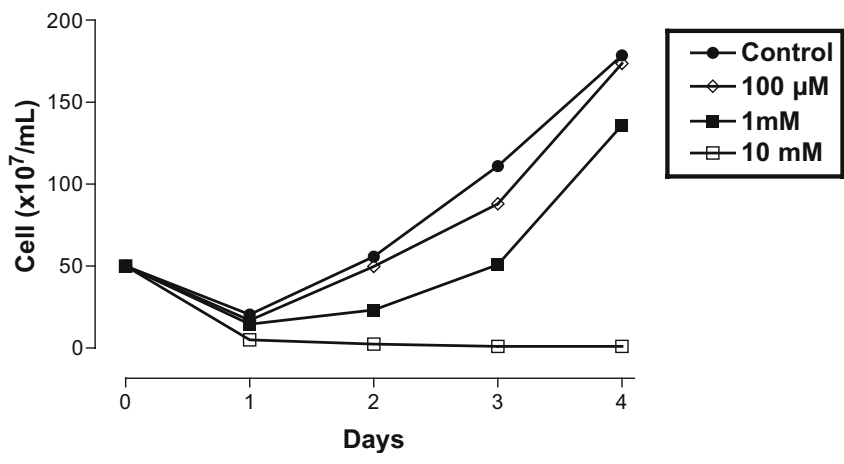

Fig. 1 Axenic proliferation of Trypanosoma cruzi epimastigotes in the absence or presence of increasing concentrations of DAB. Control (filled circle), $100 \mu \mathrm{M}$ DAB (diamond), $1 \mathrm{mM}$ DAB (filled square), $10 \mathrm{mM}$ DAB (square)

$16 \mathrm{~h}$. Pellets were solubilized in DMSO and transferred to flat-bottom 96-well microtiter plates. Formazan precipitates derived from MTT reduction were determined
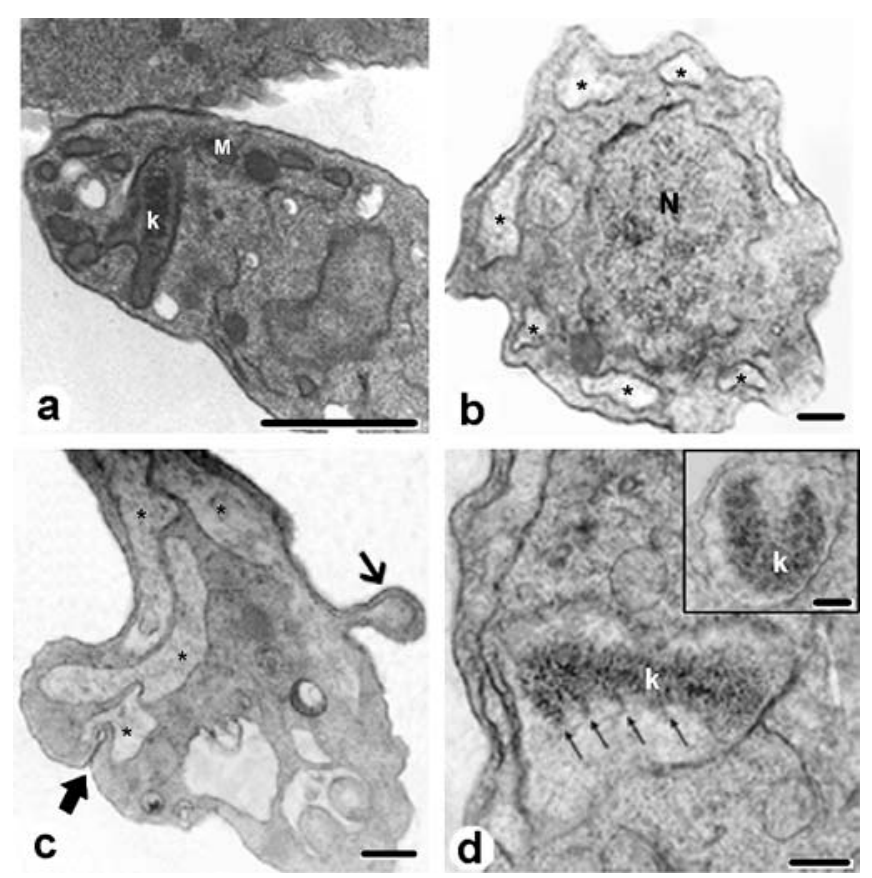

Fig. 2 Transmission electron microscopy of DAB effects on $T$. cruzi epimastigotes. a Low-magnification TEM showing the general appearance of untreated control parasites displaying normal mitochondria $(M)$ and kinetoplast $(K)$. Parasites incubated with $10 \mathrm{mM}$ $\mathrm{DAB}$ in the culture medium for $24 \mathrm{~h}$ presented remarkable mitochondrial alterations. Transversally (b) and sagitally (c) sectioned DAB-treated epimastigotes displayed reduced mitochondrial electron density all over the cells. The single mitochondrion of the parasite was remarkably damaged and washed out (asterisk). Some parasites preserved the overall cytoplasmic architecture (b), but, eventually, tubular compartments left by the mitochondrion (c) appeared to be either collapsing (wide arrow) or protruding (thin arrow) at the cell surface, drastically affecting cell ultrastructure (c). DAB treatment induced kinetoplast disorganization. The parasite kDNA $(K)$ was irregularly condensed, presenting disordered fibrils (d; arrows), or presented altered shapes (inset). a-d Bars represent $0.5,0.25,0.4$, and $0.25 \mu \mathrm{m}$, respectively 
Fig. 3 Some cells presented distended Golgi apparatus cisternae $(\mathbf{a}$; arrow). Many parasites showed numerous vesicles containing membrane-bound amorphous electron-dense materials (b; arrows), which sometimes displayed a granular appearance (arrowhead). a, b Bars represent 0.15 and $0.25 \mu \mathrm{m}$, respectively

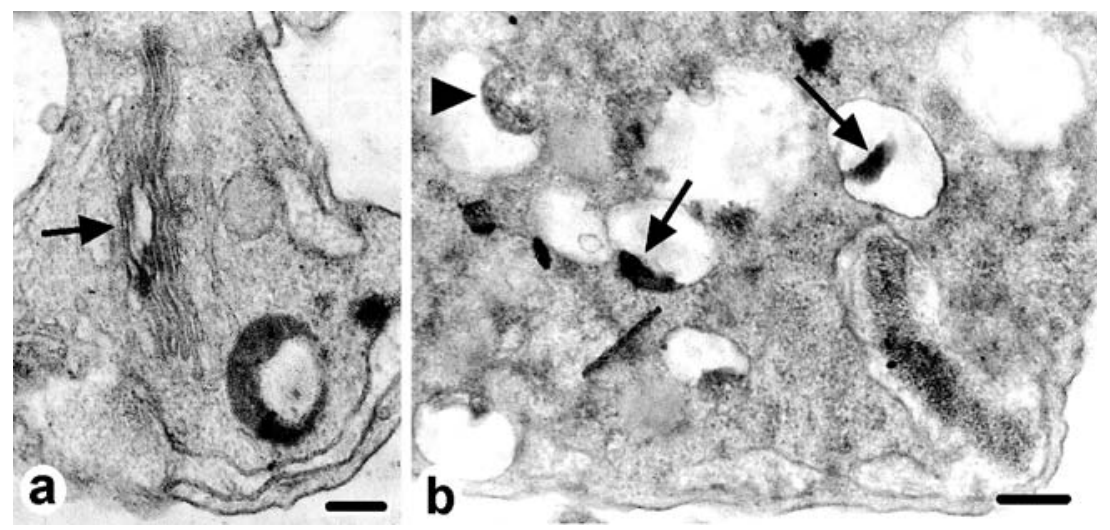

spectrophotometrically at $570 \mathrm{~nm}$. Wells containing solely MTT and DMSO were employed as controls.

Statistical analysis Presented data are representative of a minimum of three independent experiments, which yielded analogous results. Significant differences $(P<0.05$ and $P<0.001)$ were statistically analyzed using ANOVA.

\section{Results}

Proliferation of $T$. cruzi epimastigotes in vitro was reduced in a dose-dependent manner by the putrescine analogue DAB. Significant $(P<0.05)$ inhibition was achieved by $1 \mathrm{mM}$ DAB treatment (with an apparent $\mathrm{IC}_{50}$ of $1.18 \mathrm{mM}$ ) and was totally blocked by $10 \mathrm{mM}$ DAB (Fig. 1).

Transmission electron microscopy (TEM) revealed that DAB treatment resulted in profound ultrastructural changes in $T$. cruzi mitochondria, leading to a reduction of its electron density (Fig. 2a-d) as well as causing extensive damage and even complete destruction of the organelle (Fig. 2b,c). These effects ultimately resulted in a general ultrastructural disorganization (Fig. 2c). DAB also induced kinetoplast disorganization because the parasite kinetoplast DNA (kDNA) was irregularly condensed, presenting disordered kDNA fibrils (Fig. 2d, arrows), or presented altered shapes (Fig. 2, inset). Some cells presented dis-

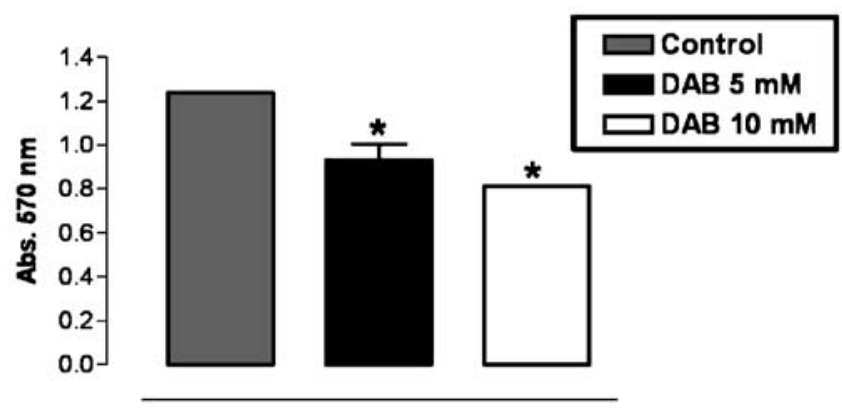

Fig. 4 Effect of DAB on mitochondrial function in T. cruzi. Epimastigotes were treated with either 5 or $10 \mathrm{mM} \mathrm{DAB}$ for $24 \mathrm{~h}$ and incubated with MTT, as described in "Materials and methods." Formazan precipitation was determined spectrophometrically at $570 \mathrm{~nm}$. Both concentrations significantly $(P<0.05)$ diminished mitochondrial activity tended Golgi apparatus cisternae (Fig. 3a) as well as numerous vesicles often containing amorphous electrondense materials that were generally bound to the lining membrane (Fig. 3b, arrows), which sometimes displayed a granular appearance (Fig. 3, arrowhead).

Since the mitochondria seem to be the main target of DAB treatment on T. cruzi epimastigotes, we next evaluated possible mitochondrial damage by MTT reduction assay. Mitochondrial function was significantly $(P<0.05)$ diminished by either 5 or $10 \mathrm{mM} \mathrm{DAB} \mathrm{(Fig.} \mathrm{4).}$

Since the mitochondria were the main sites of cellular reactive oxygen species (ROS) generation and since DAB treatment led to dramatic changes in this organelle, we decided to evaluate whether mitochondrial changes would be associated with oxidative stress in DAB-treated parasites. Measurement of TBARS revealed that DAB effects were dose-dependent. Whereas a concentration of $5 \mathrm{mM}$ only slightly enhanced lipid peroxidation, a $10-\mathrm{mM}$ concentration diminished it considerably (Fig. 5). Interestingly, coincubation of parasites with putrescine reverted the prooxidant effects of $5 \mathrm{mM} \mathrm{DAB}$ (Fig. 5).

\section{Discussion}

Devoid of de novo polyamine biosynthesis pathway, $T$. cruzi epimastigotes are obligate polyamine scavengers (Ariyanayagam and Fairlamb 1997). There is evidence that T. cruzi amastigotes and trypomastigotes, under polyamine shortage conditions, are able to synthesize putrescine from arginine via agmatine (reviewed in Peluffo et al. 2004); however, it seems that, most of the time, this protozoan relies on diamine incorporation from the hosts. The putrescine uptake by $T$. cruzi is about 50 -fold higher than that in Leishmania mexicana (González et al. 1992). Conceivably, DAB would bind to the polyamine transporter at the parasite surface and dislocate the native diamine. However, it remains to be determined whether DAB enters the cell.

To approach the mode of action of this analogue, we employed TEM of parasites before and after DAB treatment. We noticed that DAB-treated epimastigotes displayed drastically altered or destroyed mitochondria (Fig. 2). Interestingly, this analogue also affected Leishmania mitochondria 


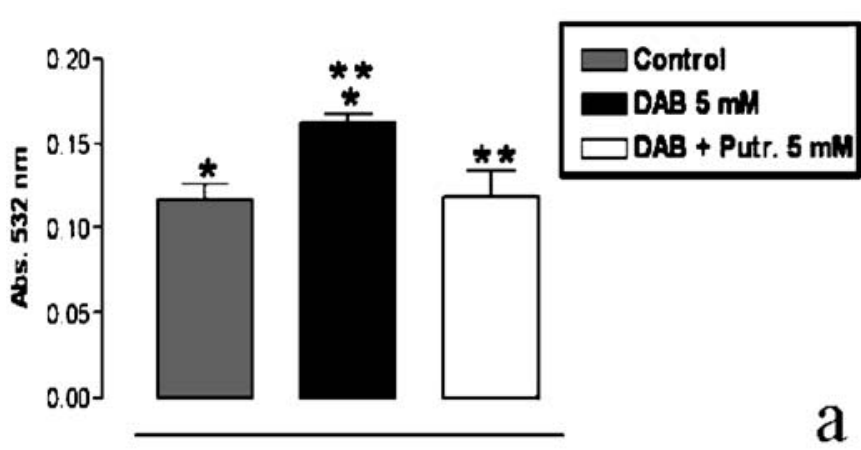

Fig. 5 Lipid peroxidation was assessed by the production of TBARS determined at $532 \mathrm{~nm}$, as described in "Materials and methods." a A concentration of $5 \mathrm{mM}$ DAB significantly $(P<0.05)$ enhanced lipid peroxidation in epimastigotes, whereas putrescine

(Valentim et al., unpublished) and redox ATP-producing trichomonad hydrogenosomes (Reis et al. 1999).

Since mitochondrial swelling is associated with reduced state 3 respiration, which may trigger permeability transition, ROS generation, and, ultimately, apoptosis, and since ATP depletion impairs both organelle swelling and procaspase activation (Robertson and Orrenius 2002), it seems reasonable that DAB-treated epimastigotes undergo necrotic death before an apoptotic process could take place. Furthermore, polyamine depletion may shift drug-induced programmed cell death to necrosis (Monti et al. 2004), and the absence of nuclear fragmentation or chromatin condensation in our experiments reinforces the inference of a necrotic process rather than an apoptotic process. Nevertheless, bis(ethyl)-polyamine analogues produced mitochondrial swelling associated with a decreased ATP content in mammalian cells (Fukuchi et al. 1992; He et al. 1994), and the organelle was severely injured (Fukuchi et al. 1992; Snyder et al. 1994). DAB-induced mitochondrial dysfunction was confirmed by MTT reduction as that in Leishmania (Valentim et al., unpublished). At advanced stages of DAB treatment, the mitochondria were somewhat enlarged, but the organelle matrix was completely destroyed. In this regard, spermine was shown to inhibit calciumand phosphate-induced mitochondrial swelling (Rigobello et al. 1993). In some parasites, mitochondrial space was either collapsed or protruded, remarkably distorting cell shapes (Fig. 2c). These morphological changes may be indicative of impaired calcium homeostasis, since sustained high cytoplasmic $\mathrm{Ca}^{2+}$ concentrations lead to cell surface blebbing by inducing cytoskeletal disorganization (Boobis et al. 1989) as well as oxidative stress in the mitochondria (Sousa et al. 2003). Since the trypanosomatid cell shape is maintained by subpellicular microtubules that run parallel underneath the plasma membrane (e.g., De Souza 2002; Vannier-Santos et al. 2002), it is reasonable to assume that parasite cytoskeleton is remarkably affected. Such calcium imbalance may be associated with the oxidation of sulfhydryl groups during oxidative stress (Boobis et al. 1989). These observations indicate that putrescine transport and, possibly, subsequent metabolism to spermidine and trypanothione are required for the maintenance of mitochon-

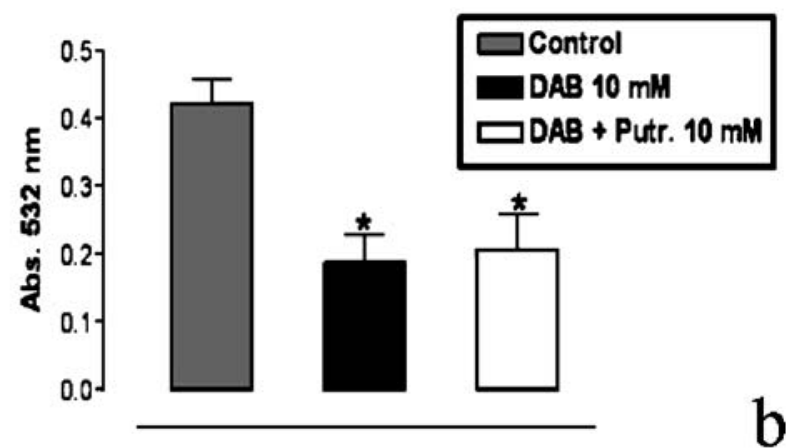

reversed it to control levels. b Parasites incubated with $10 \mathrm{mM}$ DAB presented a highly significant $(P<0.01)$ decrease in lipid peroxidation, which was not reverted by putrescine

drial structure and function as well as for cell architecture. In this regard, Giffin et al. (1986) reported that putrescine, but not spermidine, was able to stabilize $T$. brucei mitochondrial membranes. In addition, putrescine was reported to protect Escherichia coli from oxidative stress (Chattopadhyay et al. 2003; Tkachenko et al. 2001). Mitochondrial permeability transition (MPT) was shown to be involved in both necrosis and apoptosis. ROS are among the factors that trigger MPT, and polyamines may protect the mitochondria from MPT (reviewed in Fairlamb and Cerami 1992; Tassani et al. 1995).

Putrescine depletion may lead to reduced trypanothione levels and, thus, oxidative stress by ROS. T. cruzi presents a mitochondrial trypanothione-dependent peroxidase acting as an important antioxidant defense in the vicinity of the kDNA, presumably to protect the mitochondrial genome from both endogenous and exogenous oxidative stresses (Wilkinson et al. 2000).

Parasite mitochondria comprise valuable targets for chemotherapy (Kita et al. 2001), and the crystal or gentian violet used to prevent the spread of Chagas disease by blood transfusion exerts its effects on the T. cruzi mitochondria (Gadelha et al. 1989). The kDNA is the site of action of several compounds used against trypanosomatid parasites (Shapiro and Englund 1990). Benznidazole metabolites were reported to bind to nuclear and kinetoplast DNA (De Toranzo et al. 1988), producing strand breaks (Goijman et al. 1985) and extensive mitochondrial alterations in intracellular T. cruzi (Andrade and Freitas 1987). Polyamine deficiency in DAB-treated parasites could result in remarkable alterations observed in $T$. cruzi kDNA organization. This analogue may prevent putrescine uptake by the mitochondria since this organelle transports spermine, spermidine, and putrescine in mammalian cells (Toninello et al. 1992). It is noteworthy that spermine prevents glutathione release by the rat liver mitochondria and thus is presumably involved in oxidative stress defense (Toninello et al. 1992). In trypanosomatid protozoa, most thiol groups are found in $N_{1}, N_{8}$ (glutathionyl) spermidine or trypanothione (Fairlamb and Cerami 1992).

Parasitic protozoa may rely on glycolytic metabolism for energy supply for long periods, but in trypanosomatid 
parasites, the glycolytic pathway depends on the mitochondria because of a cyanide-insensitive glycerol-3phosphate oxidase system that is localized in this organelle (Clarkson et al. 1989). Therefore, mitochondria-impairing compounds may comprise important candidate agents in parasitic disease chemotherapy (e.g., Kita et al. 2001; Nihei et al. 2002). In this regard, ergosterol biosynthesis inhibitors may lead to pronounced mitochondrial damage in T. cruzi (Lazardi et al. 1990) and L. amazonensis (VannierSantos et al. 1995; Rodrigues et al. 2002). Several microbicidal agents, including nitrofurans such as nifurtimox, act as redox cycling agents, producing oxidative stress and generating $\mathrm{O}_{2}^{--}$and $\mathrm{H}_{2} \mathrm{O}_{2}$ (Docampo and Stoppani 1979; Docampo et al. 1981). The dose dependence of DAB effects may be due to the complete mitochondrial destruction observed in parasites treated with a higher concentration, since this organelle is the main source of ROS.

Enzymes such as trypanothione reductase constitute rather promising targets for chemotherapy (Austin et al. 1999; Augustyns et al. 2001). Interestingly, trypanothione is involved in the detoxification of nifurtimox and benznidazole in T. cruzi (Repetto et al. 1996). These compounds decrease glutathione and trypanothione levels in T. cruzi (Maya et al. 1997), and metabolites of nitroimidazoles, such as megazole, are efficient trypanothione scavengers (Maya et al. 2003). Thus, polyamines may play a fundamental role in $T$. cruzi resistance to the drugs of choice and may therefore provide important targets for rational drug design.

Putrescine was reported not to be required in vitro for Leishmania donovani promastigotes devoid of ODC genes. Interestingly, trypanothione concentration was reduced, although spermidine levels were relatively unaffected. Although spermidine was both required and sufficient for $L$. donovani growth, it was unable to circumvent the lethal $\Delta o d c$ mutation achieved by the addition of putrescine for both axenic growth and trypanothione production (Jiang et al. 1999). These data seem to indicate that putrescine itself may have a role in the biology of these parasites. In this regard, putrescine activates $T$. cruzi $S$-adenosylmethionine decarboxylase (Clyne et al. 2002). Furthermore, putrescine was shown to be required for the proliferation of carcinoma cells (Farriol et al. 2001) and insect neuroblasts (Cayre et al. 1997). In addition, hypoxic rat smooth muscle cells specifically require increased putrescine uptake for $\mathrm{p} 38$ mitogen-activated protein kinase activation (Ruchko et al. 2003), and this diamine can regulate the function of several genes (Pastorian et al. 2000; Fujimoto et al. 2001).

Many DAB-treated parasites displayed vesicles presenting electron-dense materials bound to the compartment membrane, resembling acidocalcisomes. Similar acidocalcisome-like compartments were observed in L. amazonensis cells incubated with ergosterol biosynthesis inhibitors. These structures presumably comprised autophagic vacuoles (Vannier-Santos et al. 1995, 1999). Some parasites presented distended Golgi apparatus cisternae. Golgi apparatus swelling and dysfunction were previously no- ticed in different polyamine-deficient mammalian cell types (Sakamaki et al. 1989).

The consumption of the arginine pool for putrescine synthesis by macrophages may be associated with reduced nitric oxide (NO) production (Mills 2001), enhancing $T$. cruzi intracellular proliferation (Freire-de-Lima et al. 2000; Piacenza et al. 2001; Peluffo et al. 2004). Similarly, T. brucei arginine uptake for polyamine synthesis reduces NO production by macrophages (Gobert et al. 2000). Therefore, antagonists of polyamine synthesis/transport may promote microbicidal activity by providing multifunctional chemotherapeutic agents.

Acknowledgements The authors thank Claudio Pereira Figueira (Centro de Pesquisas Gonçalo Moniz-FIOCRUZ) and Sebastião da Cruz (Instituto de Biofísica Carlos Chagas Filho, Universidade Federal do Rio de Janeiro) for technical assistance, Elisângela Sodré for secretarial assistance, and Hugo Vasconcelos for help with EM digital images. This work was sponsored by PROCAD-CAPES, FAPERJ, CNPq, and FIOCRUZ.

\section{References}

Andrade SG, Freitas LA (1987) Trypanosoma cruzi: cardiac myocells alterations due to spontaneous or therapeutically induced intracellular parasite disintegration. Cell Mol Biol 33:797-805

Ariyanayagam MR, Fairlamb AH (1997) Diamine auxotrophy may be a universal feature of Trypanosoma cruzi epimastigotes. Mol Biochem Parasitol 84:111-121

Arteaga-Nieto P, Villagomez-Castro JC, Calvo-Mendez C, LopezRomero E (1996) Partial purification and characterization of ornithine decarboxylase from Entamoeba histolytica. Int J Parasitol 26:253-260

Augustyns K, Amssoms K, Yamani A, Rajan PK, Haemers A (2001) Trypanothione as a target in the design of antitrypanosomal and antileishmanial agents. Curr Pharm Des 7:1117-1141

Austin SE, Khan MO, Douglas KT (1999) Rational drug design using trypanothione reductase as a target for anti-trypanosomal and anti-leishmanial drug leads. Drug Des Discov 16:5-23

Bacchi CJ, Yarlett N (2002) Polyamine metabolism as chemotherapeutic target in protozoan parasites. Mini Rev Med Chem 2:553-563

Bacchi CJ, Nathan HC, Hutner SH, McCann PP, Sjoerdsma A (1980) Polyamine metabolism: a potential therapeutic target in trypanosomes. Science 210:332-334

Boobis AR, Fawthrop DJ, Davies DS (1989) Mechanisms of cell death. Trends Pharmacol Sci 10:275-280

Calvo-Mendez C, Villagomez-Castro JC, Lopez-Romero E (1993) Ornithine decarboxylase activity in Entamoeba invadens. Int J Parasitol 23:847-852

Cayre M, Strambi C, Charpin P, Augier R, Strambi A (1997) Specific requirement of putrescine for the mitogenic action of juvenile hormone on adult insect neuroblasts. Proc Natl Acad Sci U S A 94:8238-8242

Chattopadhyay MK, Tabor CW, Tabor H (2003) Polyamines protect Escherichia coli cells from the toxic effect of oxygen. Proc Natl Acad Sci U S A 100:2261-2265

Clarkson AB Jr, Bienen EJ, Pollakis G, Grady RW (1989) Respiration of bloodstream forms of the parasite Trypanosoma brucei brucei is dependent on a plant-like alternative oxidase. J Biol Chem 264:17770-17776

Clyne T, Kinch LN, Phillips MA (2002) Putrescine activation of Trypanosoma cruzi S-adenosylmethionine decarboxylase. Biochemistry $41: 13207-13216$

Coura JR (1988) Epidemiologic determinants of Chagas' disease in Brazil: the infection, the disease and its morbidity/mortality. Mem Inst Oswaldo Cruz 83:392-402 
Coura JR, Junqueira ACV, Fernandes O, Valente SAS, Miles MA (2002) Emerging Chagas disease in Amazonian Brazil. Trends Parasitol 18:171-176

De Souza W (2002) Basic cell biology of Trypanosoma cruzi. Curr Pharm Des 8:269-285

De Toranzo EGD, Castro JA, Franke-de-Cazzulo BM, Cazzulo JJ (1988) Interaction of benznidazole reactive metabolites with nuclear and kinetoplastic DNA, proteins and lipids from Trypanosoma cruzi. Experientia 44:880-881

Docampo R, Stoppani AO (1979) Generation of superoxide anion and hydrogen peroxide induced by nifurtimox in Trypanosoma cruzi. Arch Biochem Biophys 197:317-321

Docampo R, Moreno SN, Stoppani AO, Leon W, Cruz FS, Villalta F, Muniz RF (1981) Mechanism of nifurtimox toxicity in different forms of Trypanosoma cruzi. Biochem Pharmacol 30:1947-1951

Fairlamb AH, Cerami A (1992) Metabolism and functions of trypanothione in the Kinetoplastida. Annu Rev Microbiol 46:695-729

Farriol M, Segovia-Silvestre T, Castellanos JM, Venereo Y, Orta X (2001) Role of putrescine in cell proliferation in a colon carcinoma cell line. Nutrition 17:934-938

Freire-de-Lima CG, Nascimento DO, Soares MB, Bozza PT, CastroFaria-Neto HC, Mello FG, DosReis GA, Lopes MF (2000) Uptake of apoptotic cells drives the growth of a pathogenic trypanosome in macrophages. Nature 403:199-203

Fujimoto S, Yano Y, Nishiguchi S, Koh N, Tamori A, Shiomi S, Kuroki T, Otani S (2001) Identification of genes differentially expressed by putrescine in HepG2 hepatoblastoma cells. Hepatol Res 20:207-215

Fukuchi J, Kashiwagi K, Kusama-Eguchi K, Terao K, Shirahata A, Igarashi K (1992) Mechanism of the inhibition of cell growth by $N_{1}, N_{12}$-bis(ethyl)spermine. Eur J Biochem 209:689-696

Gadelha FR, Moreno SN, De Souza W, Cruz FS, Docampo R (1989) The mitochondrion of Trypanosoma cruzi is a target of crystal violet toxicity. Mol Biochem Parasitol 34:117-126

Giffin BF, McCann PP, Bacchi CJ (1986) Effect of putrescine on the respiration of Trypanosoma brucei brucei. Mol Biochem Parasitol 20:165-171

Gobert AP, Daulouede S, Lepoivre M, Boucher JL, Bouteille B, Buguet A, Cespuglio R, Veyret B, Vincendeau P (2000) Larginine availability modulates local nitric oxide production and parasite killing in experimental trypanosomiasis. Infect Immun 68:4653-4657

Goijman SG, Frasch AC, Stoppani AO (1985) Damage of Trypanosoma cruzi deoxyribonucleic acid by nitroheterocyclic drugs. Biochem Pharmacol 34:1457-1461

González NS, Ceriani C, Algranati ID (1992) Differential regulation of putrescine uptake in Trypanosoma cruzi and other trypanosomatids. Biochem Biophys Res Commun 188:120-128

Gorla NB, Ledesma OS, Barbieri GP, Larripa IB (1988) Assessment of cytogenetic damage in chagasic children treated with benznidazole. Mutat Res 206:217-220

He Y, Suzuki T, Kashiwagi K, Kusama-Eguchi K, Shirahata A, Igarashi K (1994) Correlation between the inhibition of cell growth by bis(ethyl)polyamine analogues and the decrease in the function of mitochondria. Eur J Biochem 221:391-398

Jiang Y, Roberts SC, Jardim A, Carter NS, Shih S, Ariyanayagam M, Fairlamb AH, Ullman B (1999) Ornithine decarboxylase gene deletion mutants of Leishmania donovani. J Biol Chem 274:3781-3788

Kita K, Miyadera H, Saruta F, Miyoshi H (2001) Parasite mitochondria as a target for chemotherapy. J Health Sci 47:219-239

Lazardi K, Urbina JÁ, De Souza W (1990) Ultrastructural alterations induced by two ergosterol biosynthesis inhibitors, ketoconazole and terbinafine, on epimastigotes and amastigotes of Trypanosoma (Schizotrypanum) cruzi. Antimicrob Agents Chemother 34:2097-2105

Le Quesne AS, Fairlamb AH (1996) Regulation of a high-affinity diamine transport system in Trypanosoma cruzi epimastigotes. Biochem J 316:481-486
Marton LJ, Pegg AE (1995) Polyamines as targets for therapeutic intervention. Annu Rev Pharmacol Toxicol 35:55-91

Maya JD, Repetto Y, Agosin M, Ojeda JM, Tellez R, Gaule C, Morello A (1997) Effects of nifurtimox and benznidazole upon glutathione and trypanothione content in epimastigote, trypomastigote and amastigote forms of Trypanosoma cruzi. Mol Biochem Parasitol 86:101-106

Maya JD, Bollo S, Nuñez-Vergara LJ, Squella JA, Repetto Y, Morello A, Périé J, Chauvière G (2003) Trypanosoma cruzi: effect and mode of action of nitroimidazole and nitrofuran derivatives. Biochem Pharmacol 65:999-1006

Martinez-Pacheco M, Rodriguez G, Reyna G, Calvo-Mendez C, Ruiz-Herrera J (1989) Inhibition of the yeast-mycelial transition and the phorogenesis of Mucorales by diamino butanone. Arch Microbiol 15:110-114

Mills CD (2001) Macrophage arginine metabolism to ornithine/urea or nitric oxide/citrulline: a life or death issue. Crit Rev Immunol 21:399-425

Monti MG, Ghiaroni S, Marverti G, Montanari M, Moruzzi MS (2004) Polyamine depletion switches the form of 2-deoxy-Dribose-induced cell death from apoptosis to necrosis in HL-60 cells. Int J Biochem Cell Biol 36:1238-1248

Müller S, Coombs GH, Walter RD (2001) Targeting polyamines of parasitic protozoa in chemotherapy. Trends Parasitol 17:242249

Nihei C, Fukai Y, Kita K (2002) Trypanosome alternative oxidase as a target of chemotherapy. Biochim Biophys Acta 1587:234 239

Pastorian KE, Hawel L, Byus CV (2000) Identification of putrescine-responsive mRNAs in Chinese hamster ovary cells using representational difference analysis. Biochim Biophys Acta 1524:131-142

Pegg AE, McCann PP (1982) Polyamine metabolism and function. Am J Physiol 243:212-221

Peluffo G, Piacenza L, Irigoin F, Alvarez MN, Radi R (2004) Larginine metabolism during interaction of Trypanosoma cruzi with host cells. Trends Parasitol 20:363-369

Piacenza L, Peluffo G, Radi R (2001) L-arginine-dependent suppression of apoptosis in Trypanosoma cruzi: contribution of the nitric oxide and polyamine pathways. Proc Natl Acad Sci U S A 98:7301-7306

Reis IA, Martinez M, Yarlett N, Silva-Filho FC, Vannier-Santos MA (1999) Inhibition of polyamine synthesis arrests trichomonad growth and induces destruction of hydrogenosomes. Antimicrob Agents Chemother 43:1919-1923

Repetto Y, Opazo E, Maya JD, Agosin M, Morello A (1996) Glutathione and trypanothione in several strains of Trypanosoma cruzi: effect of drugs. Comp Biochem Physiol B Biochem Mol Biol 115:281-285

Rigobello MP, Toninello A, Siliprandi D, Bindoli A (1993) Effect of spermine on mitochondrial glutathione release. Biochem Biophys Res Commun 194:1276-1281

Robertson JD, Orrenius S (2002) Role of mitochondria in toxic cell death. Toxicology 181-182:491-496

Rodrigues JC, Attias M, Rodriguez C, Urbina JA, De Souza W (2002) Ultrastructural and biochemical alterations induced by 22,26-azasterol, a delta(24(25))-sterol methyltransferase inhibitor, on promastigote and amastigote forms of Leishmania amazonensis. Antimicrob Agents Chemother 46:487-499

Ruchko M, Gillespie MN, Weeks RS, Olson JW, Babal P (2003) Putrescine transport in hypoxic rat main PASMCs is required for p38 MAP kinase activation. Am J Physiol Lung Cell Mol Physiol 284:179-186

Sakamaki Y, Terao K, Ito E, Kashiwagi K, Igarashi K (1989) Swelling of the Golgi apparatus and decrease of galactosyltransferase in polyamine-deficient bovine lymphocytes and epithelium of mouse small intestine. Biochem Pharmacol 38:1083-1089

Shapiro TA, Englund PT (1990) Selective cleavage of kinetoplast DNA minicircles promoted by antitrypanosomal drugs. Proc Natl Acad Sci U S A 87:950-954 
Snyder RD, Beach DC, Loudy DE (1994) Anti-mitochondrial effects of bisethyl polyamines in mammalian cells. Anticancer Res 14:347-356

Sousa SC, Maciel EN, Vercesi AE, Castillo RF (2003) $\mathrm{Ca}^{2+}$-induced oxidative stress in brain mitochondria treated with the respiratory chain inhibitor rotenone. FEBS Lett 543:179-183

Tassani V, Biban C, Toninello A, Siliprandi D (1995) Inhibition of mitochondrial permeability transition by polyamines and magnesium: importance of the number and distribution of electric charges. Biochem Biophys Res Commun 207:661-667

Tkachenko A, Nesterova L, Pshenichnov M (2001) The role of the natural polyamine putrescine in defense against oxidative stress in Escherichia coli. Arch Microbiol 176:155-157

Toninello A, Dalla Via L, Siliprandi D, Garlid KD (1992) Evidence that spermine, spermidine, and putrescine are transported electrophoretically in mitochondria by a specific polyamine uniporter. J Biol Chem 267:18393-18397

Trouiller P, Olliaro P, Torreele E, Orbinski J, Laing R, Ford N (2002) Drug development for neglected diseases: a deficient market and a public-health policy failure. Lancet 359:2188-2194
Ueno Y, Fukumatsu M, Ogasawara A, Watanabe T, Mikami T, Matsumoto T (2004) Hyphae formation of Candida albicans is regulated by polyamines. Biol Pharm Bull 27:890-892

Vannier-Santos MA, Urbina JA, Martiny A, Neves A, De Souza W (1995) Alterations induced by the antifungal compounds ketoconazole and terbinafine in Leishmania. J Eukaryot Microbiol 42:337-346

Vannier-Santos MA, Martiny A, Lins U, Urbina JA, Borges VM, De Souza W (1999) Impairment of sterol biosynthesis leads to phosphorus and calcium accumulation in Leishmania acidocalcisomes. Microbiology 145:3213-3220

Vannier-Santos MA, Martiny A, De Souza W (2002) Cell biology of Leishmania spp.: invading and evading. Curr Pharm Des 8:297318

Wilkinson SR, Temperton NJ, Mondragon A, Kelly JM (2000) Distinct mitochondrial and cytosolic enzymes mediate trypanothione-dependent peroxide metabolism in Trypanosoma cruzi. J Biol Chem 275:8220-8225 